\title{
Aprendizaje y autoevaluación online de estudiantes universitarios: la matemática financiera en entornos virtuales
}

\section{J. David Cabedo Semper ${ }^{\mathrm{a}}, \mathbf{M}^{\mathrm{a}}$ Amparo Maset Llaudes $^{\mathrm{b}}$ y Nuria Segarra Adell ${ }^{\mathrm{c}}$}

${ }^{a}$ Departamento de Finanzas y Contabilidad. Universitat Jaume I. cabedo@uji.es, ${ }^{b}$ Departamento de Finanzas y Contabilidad. Universitat Jaume I. maset@uji.es y ${ }^{c}$ Departamento de Finanzas y Contabilidad. Universitat Jaume I. nsegarra@uji.es

\begin{abstract}
The course on Financial Mathematics is a compulsory course, part of the Basic Formation in three undergraduate degrees at Jaume I university: Finance and Accounting, Economics and Business Management. It is a mathematics course, with a high number of enrolled students and with a group of teachers with special characterstics. Due to this things, the implementation of an individualized monitoring system becomes complex. In this paper we present a methodological innovation proposal conducted during the school year 2014/15. The innovation consists of the virtual and formative self-assessment of three assignments. This innovation focuses on the involvement of students in a reflexive and continuous learning process. Furthermore, it pursuits a suitable and timely feedback that helps students to improve their academic performance. Our empirical results show that the virtual and formative self-assessment process has had a positive effect so in the number of students that attend the final exam as in the number of students that pass successfully the course.
\end{abstract}

Keywords: Self-assessment, formative assessment, succeed, Moodle, mathematics, assessment tools, timely feedback.

\footnotetext{
Resumen

Matemática de las Operaciones Financieras es una asignatura de Formación Básica de primer año que todos los alumnos tienen que cursar obligatoriamente en los grados universitarios de Economía, Contabilidad y Finanzas y Administración de Empresas de la Universidad Jaume I de Castellón. Por su naturaleza matemática, por el elevado número de alumnos matriculados y por las características de los profesores que imparten docencia, resulta dificil un seguimiento individualizado del aprendizaje del estudiantado. La presente comunicación expone una propuesta de innovación metodológica introducida en dicha asignatura durante el curso 2014/2015. La innovación consiste en la autoevaluación virtual formativa de los alumnos de tres casos prácticos. El objetivo es que los alumnos reflexionen sobre su aprendizaje de un modo continuo y autónomo. Además, se pretende que reciban un feedback eficaz y oportuno que les ayude a
} 


\begin{abstract}
mejorar su rendimiento académico. El análisis empírico presentado demuestra que la autoevaluación formativa virtual propuesta ha influido muy positivamente tanto en el aumento del porcentaje de alumnos que se presentan al examen final como en su nota media.
\end{abstract}

Palabras clave: Autoevaluación; evaluación formativa; herramientas de evaluación; éxito; Moodle; matemáticas; feedback oportuno.

\title{
1. Introducción
}

Varios han sido, en los últimos años, los determinantes que han provocado un rotundo cambio de orientación de la enseñanza universitaria en nuestro contexto europeo. Entre ellos podemos encontrar, por una parte, la implantación de la Declaración de Bolonia (en adelante D de B) y por otra la generalización del uso de las tecnologías virtuales en los procesos de enseñanza-aprendizaje. Dentro de las diferentes opciones que ofrece la tecnología virtual, se está demostrando en diferentes investigaciones que la autoevaluación formativa es una de las herramientas más valiosas y fructíferas a la hora de mejorar el desempeño de los estudiantes.

\subsection{La Declaración de Bolonia, el EEES y los ECTS}

Con respecto a la $\mathrm{D}$ de $\mathrm{B}$, podría decirse que ésta dio lugar a un compromiso de cada país europeo firmante para reformar su propio sistema de enseñanza de forma totalmente voluntaria, dado que dicha reforma no se impone y los estados miembros conservan todas sus competencias respecto al contenido y la organización de sus sistemas educativos. Su objetivo principal es facilitar la comparación de títulos entre los diferentes sistemas educativos europeos. Para ello, gracias a la Declaración de Bolonia en 1999 se crea el Espacio Europeo de Educación Superior (EEES), suscrito por 30 estados, incluyendo algunos no pertenecientes a la Unión Europea. A partir de este momento comienza la evaluación de las políticas educativas simultáneamente a nivel nacional, con el objetivo de crear un amplio espacio de intercambio educativo entre todos los países, fijando el horizonte temporal en el año 2015.

El objetivo último del desarrollo del EEES se apoya en el desarrollo de la convergencia en materia educativa, lo que implica que los distintos sistemas educativos deben llevar a cabo una serie de reformas enfocadas a modificar las estructuras de los estudios universitarios con el fin de que puedan aproximarse entre ellos, colaborando de esta manera en la consecución de la cohesión europea a todos los niveles, y especialmente en lo que respecta al progreso científico y técnico. Como consecuencia de ello, en España los estudios universitarios se hallan divididos actualmente en tres niveles formativos: grado, máster y doctorado, cuyos títulos tienen validez en todo el EEES, actualmente integrado por 49 países. El objetivo fundamental del EEES, pues, es facilitar la movilidad de estudiantes, profesores, investigadores y titulados entre todos los países miembros.

(cc) EY-NC-ND 2016, Universitat Politècnica de València 
La Declaración de Bolonia incluye entre sus principales objetivos el establecimiento de un sistema de créditos europeos como el ECTS (European Credit Transfer and Accumulation System o Sistema Europeo de Transferencia y Acumulación de Créditos). Hoy en día, los créditos ECTS (European Credit Transfer System) son el estándar adoptado por todas las universidades del EEES y garantizan la convergencia de los distintos sistemas europeos de educación superior. Los créditos ECTS se basan en el trabajo personal del estudiante: horas lectivas, de estudio, elaboración de trabajo y prácticas. Un crédito ECTS equivale a entre 25 y 30 horas de trabajo del estudiante. Un curso académico equivale a 60 créditos ECTS siempre que el estudiante tenga una dedicación completa".

La estructura de los estudios tiene como base el título de grado, con una duración de 240 créditos ECTS en 4 años, excepto algunos estudios de grado con regulación específica como medicina, arquitectura o ingeniería, que tienen una duración mayor.

\subsection{La Universitat Jaume I (UJI) y el EEES}

Las titulaciones que nos afectan en este estudio (los nuevos estudios de grado de la UJI en Finanzas y Contabilidad, Grado en Economía y Grado en Administración de Empresas), implantados como efecto del Plan Bolonia, se han planteado desde la perspectiva de la especialización, pero manteniendo un tronco común en las tres titulaciones en su totalidad durante los dos primeros años (120 créditos), y parcialmente en algunas asignaturas de $3^{\circ} \mathrm{y}$ $4^{\circ}$ año. El fin es que los egresados puedan desenvolverse profesionalmente en las áreas de gestión en las empresas, administraciones públicas u otro tipo de organizaciones. La elección de cada una de estas titulaciones permitirá especializarse en distintos aspectos de gestión económica y empresarial.

La UJI ha establecido que cada crédito ECTS equivale a 25 horas de trabajo del estudiante. De ellas, entre 7, 5 y 10 horas corresponden a docencia presencial (clases, prácticas, tutorías, seminarios, etc.) y el resto son horas de trabajo autónomo del alumno (trabajos, proyectos, horas de estudio, etc.). Dado pues que un ECTS se asimila a 25 horas de trabajo del/a estudiante, un curso completo requerirá una dedicación de 1.500 horas. Asimismo, al tratarse de una titulación presencial, le corresponderá un porcentaje de presencialidad por ECTS que oscila entre el $30 \%$ y el $40 \%$. Ello sitúa el máximo de horas de asistencia en 10 , de forma que son 15 el mínimo de horas de trabajo personal por cada ECTS.

\subsection{La metodología virtual y su importancia en la educación superior. La plataforma Moodle}

Para poder implementar este nuevo sistema de trabajo, la docencia en la UJI se encuentra adaptada a las nuevas tecnologías, tanto en lo que respecta al programa académico como al desarrollo de las clases, lo que permite aplicar una metodología docente diferenciada (aula virtual, equipamiento audiovisual en las aulas..., es decir, software y hardware, respectivamente).

Una de las formas más generalizadas en que se ha implementado este desarrollo tecnológico en las universidades es la apuesta por la expansión de la educación a distancia con herramientas o aplicaciones informáticas, es decir, de manera virtual, a través de las llamadas plataformas de aprendizaje on line o virtuales, llamadas también e-learning. Este

(cc) EY-NC-ND 2016, Universitat Politècnica de València

Congreso In-Red (2016) 
tipo de plataformas se conocen como LCMS (Learning Content Management System), siendo la plataforma Moodle la utilizada por la UJI.

Una característica muy importante de esta plataforma y que resulta básica en el desarrollo y evaluación de la asignatura que estamos analizado en el presente trabajo, es que permite a los estudiantes la conversación privada, lo que lleva a la posibilidad de realizar evaluaciones individuales, corregirlas (con distintos métodos) y hacer observaciones y comentarios sobre las respuestas proporcionadas por los alumnos.

Concretamente, encontramos el llamado "Taller", actividad o recurso que puede usarse con el fin de interactuar el profesorado con los alumnos desde el punto vista de la evaluación de la asignatura, El taller es una actividad que permite la recopilación, revisión y evaluación por pares del trabajo de los estudiantes, que pueden enviar cualquier contenido digital (archivos) o escribir el texto directamente en un campo.

Los envíos son evaluados empleando un formato de evaluación de criterios múltiples definido por el profesor. El proceso de revisión por pares y el formato para comprender cómo funciona la evaluación se pueden practicar por anticipado con envíos de ejemplo proporcionados por el profesor, junto con una evaluación de referencia.

Esta actividad es la utilizada en el análisis que se presenta en esta comunicación, dado que se trata de estudiar cuáles son las repercusiones sobre los resultados de la evaluación de la asignatura MOF por parte de los alumnos según hayan hecho empleo de esta herramienta o no y, en caso afirmativo, dependiendo a su vez de en qué medida ese uso de la herramienta haya sido o no correcto.

\subsection{La relevancia de la autoevaluación formativa virtual en contextos universitarios}

Como Miguel Martínez comenta en su prólogo al artículo de Gibbs y Simpson (2009), los alumnos necesitan información estratégica y relevante para su aprendizaje, recibida oportunamente. También les favorece mucho una relación lo más estrecha posible con sus profesores, información sobre su proceso de aprendizaje y cómo optimizarlo. Todo ello puede ser mucho más accesible con las tecnologías de la información a nuestro alcance.

Por otra parte, Gibbs y Simpson (2009) comentan la importancia que tiene la evaluación en el modo en que los estudiantes se comportarán, distribuirán sus esfuerzos y aprenderán. En esta línea, Gibbs y Simpson mencionan varios estudios que demuestran que el aprendizaje es mayor si se distribuye la nota final entre exámenes y otras tareas asignadas de modo continuado durante el curso. Además, las calificaciones y trabajos eran mejores predictores de aprendizaje a largo plazo y en profundidad que los exámenes.

Por su parte, Hattie (1987) hizo un estudio en profundidad que concluyó que el factor individual más importante para conseguir buenos resultados de aprendizaje era el feedback (Gibbs y Simpson, 2009:19). Sin embargo, en educación superior con el aumento del tamaño de los grupos, dicho feedback había disminuido mucho.

Gibbs y Simpson (2009) exponen una serie de condiciones para influir en el volumen, la selección y la calidad de lo estudiado a través de la retroalimentación. Respecto al volumen, proponen ofrecer a los estudiantes suficientes actividades evaluables para ocupar suficiente

(c) EY-NC-ND 2016, Universitat Politècnica de València 
tiempo de estudio. Además dichas actividades deben enfocar a los estudiantes en lo más relevante de la asignatura. El feedback debe ser con suficiente detalle y frecuencia, oportuno y centrado en el trabajo realizado y en los criterios de evaluación señalados. El feedback es tenido en cuenta por el estudiante y le lleva a actuar.

Los hallazgos de las investigaciones y propuestas de Gibbs y Simpson han sido tenidas en cuenta en el diseño de la innovación propuesta en este trabajo. La autoevaluación formativa usando plataformas virtuales ayuda a reunir las condiciones propuestas por dichos autores.

En el presente trabajo se analiza la efectividad y la efíciencia en la implantación de la autoevaluación virtual en la asignatura de Matemáticas de las Operaciones Financieras (en adelante, MOF) mediante la comprobación de sus efectos sobre una serie de variables entre las que destacan el porcentaje de presentados al examen final y la nota final de la asignatura. La estructura del resto del trabajo es la siguiente: en la segunda sección se delimitan los objetivos. La sección tercera se dedica a la descripción de la innovación introducida. La cuarta a mostrar los resultados alcanzados. Y la última de las secciones recoge las principales conclusiones del trabajo.

\section{Objetivos}

El objetivo del presente trabajo es valorar la implementación de un sistema de evaluación continua, en le curso 2014/15, en la asignatura de MOF (asignatura de formación básica del primer curso, que es común para los grados de Economía, Administración y Dirección de Empresas, y Finanzas y Contabilidad de la Universidad Jaume I) que:

1) utilice instrumentos basados en la formación más que en la calificación;

2) implique a los estudiantes en un proceso de aprendizaje reflexivo y continuo mediante la autoevaluación;

y 3) utilice técnicas de seguimiento individualizado que proporcionen un feedback eficaz y oportuno en el tiempo en grupos con un elevado número de estudiantes.

\section{Desarrollo de la innovación}

\subsection{La asignatura}

Como ya se ha indicado anteriormente, la innovación que se presenta en esta comunicación se llevó a cabo en el curso 2014/15 en la asignatura MOF. Se trata de una asignatura de 6 créditos que, en la Universitat Jaume I, se imparte en el segundo semestre del primer curso de los grados universitarios de "Finanzas y Contabilidad", "Economía" y "Administración de Empresas". Es una asignatura de formación básica, programada en base a 50 horas presenciales distribuidas según el cronograma que muestra la figura 1:

Enseñanzas teóricas (20 horas)

\begin{tabular}{c|c|c|c|c|c|c|}
$\begin{array}{c}\text { Enseñanzas prácticas - } \\
\text { problemas ( } 10 \text { horas })\end{array}$ & Enseñanzas prácticas - laboratorio (15 horas) & $\begin{array}{c}\text { Tutorías } \\
(1 \text { hora })\end{array}$ & $\begin{array}{c}\text { Evaluación } \\
\text { ( } 4 \text { horas })\end{array}$ \\
\hline
\end{tabular}

Fig. 1 Cronograma de horas presenciales para la asignatura matemática de las operaciones financieras

(cc) EY-NC-ND 2016, Universitat Politècnica de València

Congreso In-Red (2016) 
Tal y como se puede apreciar en esta figura, las enseñanzas de tipo práctico se dividen en "problemas" y "laboratorio". En estas últimas, la clase se imparte en un aula con ordenadores y en la resolución de los problemas se emplean herramientas informáticas.

Para cada una de las modalidades de clase presencial el estudiante queda adscrito a un grupo. Estos grupos son de gran dimensión (de unos 80 alumnos en la mayoría de los casos) para las enseñanzas teóricas; de una dimensión intermedia (unos 40 estudiantes en la mayoría de los casos) para las clases de problemas; y de una dimensión más reducida (unos 20 alumnos) para las clases de laboratorio.

Al tratarse de una asignatura común para el primer curso de tres grados universitarios con elevada demanda, el número de alumnos que la cursan es grande. De hecho, para el curso 2014/15 había matriculados un total de 592 alumnos. Este elevado número de estudiantes, unido a la subdivisión de grupos que requieren las distintas modalidades de clase presencial, motiva que el número de grupos de la asignatura sea a su vez muy elevado. En concreto para el curso 2014/15 la asignatura contaba con:

- 9 grupos de teoría (incluyendo un grupo en el que la docencia se imparte íntegramente en inglés, con baja matrícula)

- 16 grupos de práctica-problemas (incluyendo un grupo en el que la docencia se imparte íntegramente en inglés, con baja matrícula)

- 32 grupos de práctica-laboratorio (incluyendo un grupo en el que la docencia se imparte íntegramente en inglés, con baja matrícula)

Este elevado número de grupos requiere a su vez la participación de un elevado número de profesores. En concreto, en el curso 2014/15 impartieron clase en alguno de los grupos un total de 17 profesores. Por cuestiones de organización docente, algunos de estos profesores impartieron sólo clases de prácticas-problemas, otros sólo clases de prácticas-laboratorio, mientras que otros impartieron las tres modalidades de clase pero a diferentes grupos de alumnos. Esto es, un profesor que impartía la teoría a un grupo de alumnos no necesariamente impartía la práctica a los mismos alumnos. A esto hay que añadir que la mayoría de los profesores de la asignatura son profesores a tiempo parcial, es decir, compatibilizan una actividad profesional externa con la docencia en la universidad, lo cual condiciona en gran medida el tiempo que pueden dedicar a esta última. El elevado número de profesores asociados con los que actualment cuenta el departamento, con una baja dedicación en créditos que se debe ajustar a grupos de docència, y el solapamiento de horarios entre grupos motiva que el profesorado de la assignatura difícilmente pueda distribuirse de un modo muy diferente al actual. Por último, aunque no por ello menos importante, debe subrayarse que la asistencia a las clases es totalmente voluntaria y que la Universitat Jaume I no tiene (ni tampoco proyecta implantar en el futuro) un sistema que permita llevar un seguimiento de la asistencia de los alumnos.

Estos datos, por sí solos, ponen de manifiesto la dificultad que comporta realizar un seguimiento individualizado de los alumnos que les proporcione un feedback oportuno sobre su grado de progreso en la asignatura. Este feedback resulta especialmente relevante para las asignaturas del campo de las matemáticas, en las que, habitualmente, los 
contenidos de los temas se asientan en conocimientos, conceptos, destrezas y habilidades adquiridos en temas anteriores. Las carencias en el dominio de estos últimos conlleva una alta probabilidad de fracaso en la consecución de los objetivos formativos de la asignatura, por lo que un adecuado feedback sobre el grado de progreso resulta de gran utilidad.

Con respecto a la evaluación, la guía docente de la asignatura prevé la realización de un examen escrito (test, desarrollo y/o problemas) que pondera un 70\% sobre la calificación final. El 30\% restante se obtiene de las actividades llevadas a cabo por los estudiantes a lo largo del semestre.

En cursos anteriores, dichas actividades consistían en la resolución, por parte del estudiante, de tres casos prácticos. El estudiante disponía de varias semanas para la resolución de estos casos, cuyo enunciado conocía, además, a priori. La resolución presentada a través de la plataforma Moodle la corregía un profesor, y el estudiante recibía la notificación con su calificación. Cada profesor corregía y calificaba los ejercicios cuando podía (recuérdese que muchos de los profesores lo son a tiempo parcial) por lo que para muchos alumnos la corrección y calificación se demoraba, en ocasiones, más de un mes. Debe remarcarse que cuando finalizaba el plazo de entrega, se publicaba en la plataforma Moodle la solución sugerida para el caso práctico correspondiente. El consultar o no dicha solución era potestativo del estudiante.

\subsection{La autoevaluación de los estudiantes}

El procedimiento de evaluación de las actividades llevadas a cabo de forma autónoma por los estudiantes que se utilizaba en cursos anteriores permitía obtener una calificación para esa parte de la asignatura, pero no reportaba beneficio adicional alguno para el alumno. A pesar de que la resolución del caso práctico estaba accesible a través de la plataforma, la voluntariedad de su consulta y la demora en las correcciones provocaban que la resolución de dichos casos prácticos no fuesen una fuente de feedback que funcionase de forma eficiente para los estudiantes Este es el motivo por el cual, ya en el curso 2013/14 se comenzó a reflexionar sobre la necesidad de introducir en la asignatura nuevos métodos de enseñanza a través del e-learning que consiguiesen implicar a los estudiantes en un proceso de aprendizaje reflexivo y continuo, utilizando instrumentos de evaluación basados en la formación más que en la calificación, e implementando técnicas de seguimiento individualizado que proporcionasen un feedback eficaz y oportuno en el tiempo. En este curso 2013/14 se llevó a cabo un proyecto piloto, en el que participaron algunos de los grupos de la asignatura en los que impartían docencia los autores del presente trabajo (sobre los resultados del proyecto piloto se puede consultar Cabedo et al., 2015 a y b). Dicho proyecto piloto sirvió de base para el diseño e implementación de la acción innovadora que se describe a continuación, que se llevó a cabo en el curso 2014/15.

En este curso 2014/15, la parte de la calificación que no proviene del examen final escrito también se obtiene a partir de la resolución de tres casos prácticos que los estudiantes presentan a través del aula virtual. No obstante, en el proceso de resolución y presentación de los casos prácticos se introducen algunas novedades. Concretamente, se da a los estudiantes un período de 48 horas para la resolución de cada caso práctico, cuyo enunciado

(c)) EY-NC-ND 2016, Universitat Politècnica de València

Congreso In-Red (2016) 
no se conoce a priori. Las fechas de resolución de los tres casos prácticos son comunes para todos los grupos (en cursos anteriores, en la medida en que se conocía el enunciado, las fechas no coincidían; cada grupo resolvía el caso práctico cuando se había impartido la materia necesaria para su resolución). Y estas fechas son conocidas por los estudiantes a principio de curso, de modo que puedan planificarse.

Ahora bien, el punto en el cual se incorpora realmente una acción innovadora es en la autocorrección del caso práctico, que se realiza del siguiente modo:

- En cuanto finaliza el período de presentación de la resolución del caso práctico, se publica en la plataforma la solución de dicho caso, y se abre otro período de 48 horas para que los estudiantes puedan autocorregir su resolución.

- Para esta autocorrección se diseña una rúbrica (una para cada caso práctico) en la que se identifican los distintos ítems que permiten llegar a la solución del caso práctico y en la que a cada uno de ellos se le asigna una ponderación en la puntuación final. La utilización de esta herramienta en la corrección permite al estudiante no sólo identificar aquello que está bien o está mal sino determinar qué es lo que se espera, cuál es el resultado al que se quiere llegar en cada una de las fases o ítems en los que se subdivide la resolución del caso práctico.

- La autocorrección que llevan a cabo los estudiantes es ciega en cuanto a puntuación. Esto significa que el estudiante identifica si la solución que ha aportado para el ítem correspondiente es la correcta, pero, en este momento, desconoce la calificación de su actividad. Lo que se pretende es introducir es un instrumento de evaluación basado en la formación más que en la calificación

- Se exige al estudiante que reflexione sobre los ítems en los que la solución aportada no coincide con la de la resolución del caso práctico facilitada por el profesorado. Se le pide al estudiante que detalle los aspectos concretos del ítem que son incorrectos y que los compare con la solución correcta. Estos comentarios son quizá los más importantes del proceso de autocorrección en la medida en que permiten determinar si, con esta autocorrección, el estudiante ha recibido un feedback adecuado y oportuno.

- El estudiante cuenta con un estímulo positivo a la hora de llevar a cabo la autocorrección del caso práctico, ya que el $20 \%$ depende precisamente de la autocorrecciòn que realice.

- Finalizado el período de autocorrección, el profesor la revisa y:

- Si la considera correcta le da el OK y publica la nota de la actividad $(80 \%)$ y la nota de la aucocorrección (que, al ser correcta, será de 20 puntos sobre 100)

- Si la considera incorrecta, rectifica la nota de la actividad, rectifica la nota de la autocorrección y las publica.

- Si el estudiante no se ha autocorregido, el profesor corrige la actividad, la puntúa y publica la calificación. 
Esta etapa del proceso permite identificar a aquellos estudiantes que se han autocorregido correctamente, o, lo que es lo mismo, que han comprendido todos aquellos ítems en los que la solución que habían aportado no es la correcta.

- Para llevar a cabo el proceso anteriormente descrito se utilizó la herramienta "Taller", incluida en la plataforma Moodle. La utilización de esta herramienta por parte de los estudiantes es muy intuitiva, ya que desde su óptica, apenas difiere de otras que incorpora Moodle. Sin embargo, la programación de la actividad de resolución de casos prácticos, la introducción de las rúbricas y la corrección tanto de lo presentado por los estudiantes como de la autocorrección que en su caso hayan realizado, requiere una formación especial por parte del profesorado. Por este motivo, antes del inicio de las clases todos los profesores de la asignatura asistieron a un curso de formación específico en esta herramienta.

En definitiva, con la innovación docente descrita en este apartado y que se ha puesto en práctica en el curso 2014/15 se pretende que el estudiante realice un seguimiento continuado de la asignatura, seguimiento que, como se ha señalado, resulta clave en las asignaturas del campo de las matemáticas en las que los conocimientos y habilidades se asientan en los adquiridos anteriormente. Esto se pretende conseguir a través de un estímulo positivo para que el alumno reflexione en torno a los ítems clave de la materia, incluidos en las rúbricas a utilizar en la autocorrección de los casos prácticos resueltos a lo largo del curso. Con ello se espera, en última instancia, una mejora en sus resultados académicos.

\section{Resultados}

\subsection{Los alumnos de la asignatura}

Como se ha comentado anteriormente, la asignatura MOF se imparte en el primer curso y es una asignatura de las llamadas de formación básica (un $90 \%$ de los 60 créditos ECTS que se imparten en el primer curso pertenecen a este tipo, el resto se trata de asignaturas obligatorias), común para los grados de Administración de Empresas (ADE), Economía (ECO) y Finanzas y Contabilidad (FICO). La figura 2 recoge la distribución de alumnos por grados. Tal y como se puede apreciar en esta figura 2 la mayor parte de alumnos corresponden a los grados de Finanzas y Contabilidad y de Administración de Empresas.

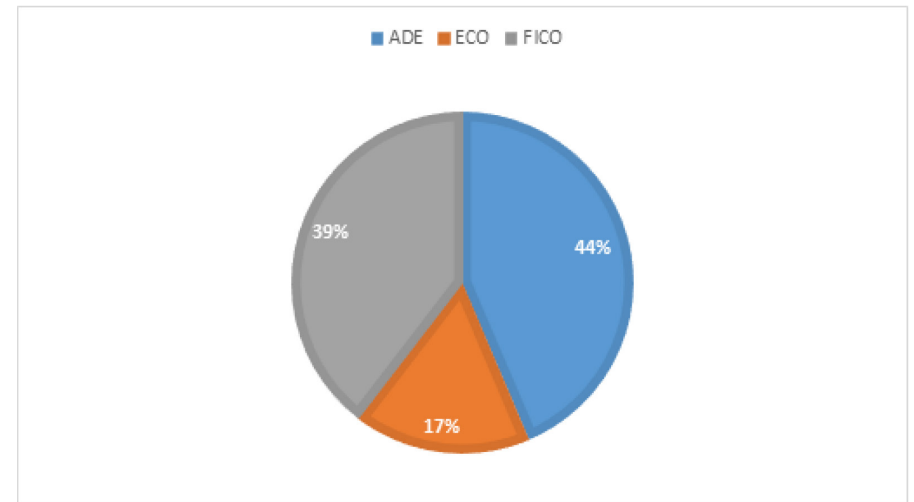

Fig. 2 Alumnos matriculados en MOF por grado. Curso 2014/15

(c)) EY-NC-ND 2016, Universitat Politècnica de València

Congreso In-Red (2016) 
Por su parte la figura 3 recoge la distribución de los estudiantes de la asignatura en función de las convocatorias consumidas antes de realizarse el primero de los exámenes del curso 2014/15. Tal y como se puede apreciar en esta figura 3 el 25\% de los estudiantes repetía la asignatura y, de ellos, la gran mayoría llevaba consumidas 3 o menos convocatorias.

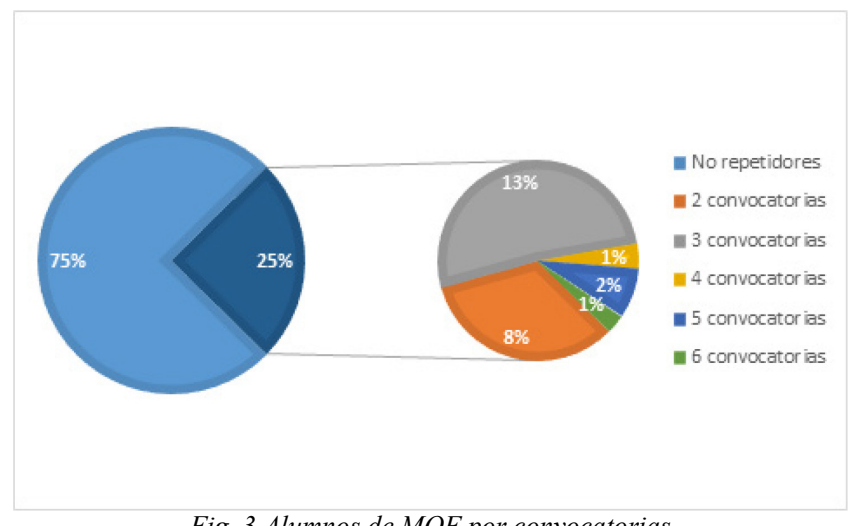

Fig. 3 Alumnos de MOF por convocatorias

\subsection{Resultados de la innovación introducida}

Con la finalidad de determinar si la la innovación descrita en el apartado anterior ha tenido o no efectos positivos, se han analizado los resultados obtenidos por los estudiantes de la asignatura, comparando los alcanzados por aquellos que han llevado a cabo de forma adecuada el proceso de autocorrección de casos prácticos diseñado con los de aquellos otros que no lo han hecho. Tal y como se ha descrito anteriormente, el $20 \%$ de la calificación de estos casos prácticos correspondía al proceso de autocorrección: si el alumno se había autocorregido según las pautas establecidas, cuando el profesor revisaba la autocorrección, le mantenía dicha clasificación. En otro caso, no. De este modo, se ha considerado que un estudiante ha llevado a cabo de forma adecuada el proceso diseñado de autocorrección de casos prácticos (grupo objetivo: ha participado en la innovación) si ha obtenido esta calificación máxima del $20 \%$ en dos de los tres casos prácticos planteados a lo largo del semestre. En otro caso, se ha considerado que el estudiante no ha llevado de forma adecuada el proceso de autocorrección (grupo de control: no ha participado en la innovación). Debe subrayarse que se ha considerado que no han participado en la innovación aquellos alumnos que, habiéndose autocorregido, no lo han hecho adecuadamente porque en el aula virtual se les han facilitado modelos suficientes sobre cómo era una correcta autocorrección (detalle de los comentarios, contraste de la respuesta correcta modelo y la propia con errores, explicación de la base teórica que justificaba la respuesta correcta y descartaba la incorrecta, etc.). Asimismo, se les ha facilitado modelo de cómo era una autocorrección no adecuada (ponerse bien cuando estaba mal o ponerse mal pero sin comentarios, sin explicar el porqué). Aquellos alumnos que, teniendo los dos modelos de autocorrección adecuada y no adecuada, habían usado el de la no adecuada, han sido excluidos. En todo caso, dado que es posible que algún alumno no haya entendido bien el proceso, en una primera instancia, y que, por tanto, sea este el motivo de no haberse corregido bien uno de los casos prácticos, se ha considerado que han participado en la 
innovación aquellos estudiantes que se han autocorregido bien al menos dos de los tres casos prácticos.

Con la anterior premisa, el análisis de los resultados se ha centrado en tres aspectos: en el abandono/permanencia de los estudiantes, en el éxito a la hora de superar la asignatura y en la calificación que han obtenido en el examen. A continuación se detalla el análisis llevado a cabo en cada uno de estos puntos.

\subsubsection{Abandono/permanencia}

El abandono/permanencia del estudiante se ha medido en función de su presentación o no presentación al examen final, obligatorio para superar la asignatura. De este modo, para comprobar si, en este punto, hay diferencias entre los estudiantes del grupo objetivo y del de control, se ha contrastado si existen diferencias estadísticamente significativas entre la proporción de estudiantes de este último grupo que se ha presentado al examen de cualquiera de las dos convocatorias ordinarias de la asignatura y la proporción de estudiantes del grupo de control que se ha presentado al mencionado examen. Para ello se utiliza el test de la chi-cuadrado. Los resultados obtenidos se muestran en la tabla 1.

Tabla 1. Contraste de la Chi-cuadrado sobre la proporción de alumnos presentados

\begin{tabular}{|l|c|c|c|}
\cline { 2 - 4 } \multicolumn{1}{c|}{} & Grupo Objetivo & Grupo de Control & Total \\
\hline $\begin{array}{l}\text { Número de alumnos } \\
\text { presentados }\end{array}$ & 212 & 210 & 422 \\
\hline $\begin{array}{l}\text { Número de alumnos no } \\
\text { presentados }\end{array}$ & 13 & 157 & 170 \\
\hline $\begin{array}{l}\text { Proporción de alumnos } \\
\text { presentados sobre el total }\end{array}$ & 0,942 & 0,572 & 0,713 \\
\hline
\end{tabular}

\begin{tabular}{|c|r|c|}
\hline \multicolumn{3}{|c|}{ Contraste de hipótesis } \\
\hline Intervalo de confianza al 95\% & $\begin{array}{c}\text { Estadístico } \\
\text { Chi-cuadrado }\end{array}$ & p-valor \\
\hline $0,3201,000$ & 93,2928 & $<2.2 \mathrm{e}-16$ \\
\hline
\end{tabular}

Hipótesis nula: La proporción de estudiantes presentados es la misma en el grupo objetivo y en el de control. Hipótesis alternativa: La proporción de estudiantes del grupo objetivo que se han presentado al examen es mayor que la del grupo de control.

Tal y como puede apreciarse en la tabla 1 , a un $5 \%$ de significatividad se rechaza la hipótesis nula contrastada (el p-valor es menor que el mencionado umbral). Por lo tanto se puede afirmar que la proporción de alumnos que han participado en la innovación y se han presentado al examen final es mayor que la proporción de alumnos que no han participado en la innovación y se han presentado al examen final. Esto es, el participar en la innovación puede tener una influencia positiva, en la medida en que la tasa de abandono de la asignatura en los alumnos del grupo objetivo es inferior.

\subsection{2. Éxito}

El segundo de los resultados que se ha analizado es el éxito del estudiante en la asignatura. Se considera que un estudiante tiene éxito en la asignatura si la supera en cualquiera de las

(cc) EY-NC-ND 2016, Universitat Politècnica de València

Congreso In-Red (2016) 
dos convocatorias ordinarias del curso. En este punto debe subrayarse que un estudiante supera la asignatura si cumple con dos condiciones:

- Obtener más de un $40 \%$ de la puntuación máxima del examen final

- Superar el umbral del $50 \%$ con la suma de la calificación obtenida en el examen final y la obtenida en las actividades realizadas a lo largo del curso.

Es decir, se exige una nota mínima en el examen presencial para que puedan ser computadas las calificaciones obtenidas en las actividades no presenciales. Esta medida está encaminada a superar los potenciales ardides no lícitos que los estudiantes hayan podido utilizar en la resolución y autocorrección de las actividades no presenciales.

La tabla 2 recoge los resultados obtenidos en relación con la proporción de estudiantes que aprueban la asignatura.

Tabla 2. Contraste de la Chi-cuadrado sobre la proporción de alumnos aprobados

\begin{tabular}{|l|c|c|c|}
\cline { 2 - 4 } \multicolumn{1}{c|}{} & Grupo Objetivo & Grupo de Control & Total \\
\hline $\begin{array}{l}\text { Número de alumnos } \\
\text { presentados y aprobados }\end{array}$ & 151 & 94 & 245 \\
\hline $\begin{array}{l}\text { Número de alumnos } \\
\text { presentados y no aprobados }\end{array}$ & 61 & 116 & 177 \\
\hline $\begin{array}{l}\text { Proporción de alumnos } \\
\text { aprobados sobre presentados }\end{array}$ & 0,712 & 0,448 & 0,581 \\
\hline
\end{tabular}

\begin{tabular}{|c|r|c|}
\hline \multicolumn{3}{|c|}{ Contraste de hipótesis } \\
\hline Intervalo de confianza al 95\% & $\begin{array}{c}\text { Estadístico } \\
\text { Chi-cuadrado }\end{array}$ & p-valor \\
\hline $0,1881,000$ & 30,343 & $1,81 \mathrm{E}-08$ \\
\hline
\end{tabular}

Hipótesis nula: La proporción de estudiantes presentados y aprobados es la misma en el grupo objetivo y en el de control. Hipótesis alternativa: La proporción de estudiantes del grupo objetivo que se han presentado y aprobado el examen es mayor que la del grupo de control.

Tal y como puede apreciarse en la tabla 2 , con un $5 \%$ de significatividad, se rechaza la hipótesis nula contrastada (el p-valor es menor que el mencionado umbral). Por lo tanto se puede afirmar que la proporción de alumnos que han participado en la innovación, se han presentado al examen final y han aprobado es mayor que la proporción de alumnos que no han participado en la innovación, se han presentado al examen final y lo han aprobado. Esto es, el participar en la innovación puede influir positivamente en el éxito del estudiante.

\subsubsection{Nota en el examen final}

El tercero de los resultados que se ha analizado es la nota obtenida por el estudiante en la prueba presencial. Lo que se pretende determinar es si la participación en la innovación influye en las habilidades y conocimientos demostrados en una prueba de evaluación presencial homogénea para todos los estudiantes. Para ello se han analizado las calificaciones medias obtenidas en el examen presencial (recuérdese que la máxima calificación posible en esta prueba es de 7 puntos) por los estudiantes del grupo objetivo que han superado la asignatura y por los estudiantes del grupo de control que también han

(cc) EY-NC-ND 2016, Universitat Politècnica de València 
superado la asignatura. El método elegido para comparar dichas medias es un test basado en la $t$ de Student. Concretamente se ha utilizado el test de Welch que es el apropiado cuando no existen razones para suponer que las varianzas en ambos grupos (las varianzas de las calificaciones de los alumnos presentados y aprobados) son iguales. De hecho, y previamente a la realización de este test, se ha contrastado la hipótesis nula de igualdad de varianzas entre las notas de los estudiantes de ambos grupos, utilizando un test basado en la distribución F de Snedecor. El resultado ha sido el de rechazar la hipótesis nula planteada, con un nivel de significación inferior al 5\%.

La tabla 3 recoge los resultados obtenidos para el test de Welch.

Tabla 3. Contraste de Welch sobre la nota media de los alumnos aprobados

\begin{tabular}{|l|c|c|}
\cline { 2 - 3 } \multicolumn{1}{c|}{} & Grupo Objetivo & Grupo de Control \\
\hline $\begin{array}{l}\text { Número de alumnos } \\
\text { presentados y aprobados }\end{array}$ & 151 & 94 \\
\hline $\begin{array}{l}\text { Nota media de los alumnos } \\
\text { presentados y aprobados }\end{array}$ & 4,57 & 4,18 \\
\cline { 2 - 3 } & \multicolumn{2}{|c|}{ Contraste de hipótesis } \\
\cline { 2 - 3 } & $\begin{array}{c}\text { Estadístico } \\
\text { de Welch }\end{array}$ & p-valor \\
\cline { 2 - 3 } & $-3,063$ & $1,22 \mathrm{E}-03$ \\
\hline
\end{tabular}

Hipótesis nula: La nota media de los estudiantes presentados y aprobados es la misma en el grupo objetivo y en el de control. Hipótesis alternativa: La nota media de los estudiantes presentados y aprobados es mayor en el grupo objetivo que en el de control

Tal y como puede apreciarse en la tabla 3 , con un $5 \%$ de significatividad, se rechaza la hipótesis nula contrastada (el p-valor es menor que el mencionado umbral). Es decir, el haber participado en la innovación no solo aumenta la probabilidad de aprobar sino que también mejora la nota del examen. Este resultado es el que cabría esperar, en la medida en que una de las finalidades de la innovación introducida era la de incentivar el estudio de forma continuada por parte de los estudiantes, lo que, para una asignatura del campo de la matemática, en última instancia, debería reflejarse en una mejora en el rendimiento.

\section{Conclusiones}

En el presente trabajo se exponen los resultados de un proyecto de innovación centrado en reforzar el autoaprendizaje en la asignatura de Matemática de las Operaciones Financieras (Empresa), asignatura de impartición obligatoria, de primer curso, común en los grados del área económica que actualmente se imparten en la Universitat Jaume I de Castellón. En el desarrollo del proyecto se han conformado dos grupos de estudiantes: aquellos que han utilizado activamente la innovación introducida (grupo objetivo) y aquellos que no lo han hecho (grupo de control).

Un primer resultado que se ha observado es que la participación en la innovación tiene un resultado positivo, en la medida en que reduce la tasa de abandono: el 94\% de estudiantes del grupo objetivo (aquélllos estudiantes que han utilizado activametne la innovación

(cc) EY-NC-ND 2016, Universitat Politècnica de València

Congreso In-Red (2016) 
introducida) se presentó al examen final (esto es, la tasa de abandono fue del 6\%), frente al $57 \%$ en el grupo de control (aquélllos estudiantes que no han utilizado activametne la innovación introducida). En este último grupo, por tanto, la tasa de abandono fue del 43\%). La diferencia entre ambas proporciones es estadísticamente significativa, de acuerdo con el test de la chi-cuadrado.

Otro resultado interesante hace referencia a la tasa de éxito: más de un $71 \%$ de los estudiantes del grupo objetivo aprobaron la asignatura, frente a tan solo un $44 \%$ en el grupo de control (la diferencia entre ambas proporciones es estadísticamente significativa, de acuerdo con el test de la chi-cuadrado). Es más, se ha constatado que la participación en la innovación no sólo aumenta la probabilidad de superar la asignatura, sino que mejora el rendimiento: la nota media obtenida en el examen presencial es mayor para los estudiantes del grupo objetivo.

En resumen, la innovación introducida ha sido eficaz y sería altamente recomendable profundizar más en la misma línea de trabajo con el objetivo de mejorar los resultados alcanzados.

\section{Referencias}

Benito, A. (2005). Nueva claves para la docencia universitaria. Madrid: Narcea

Cabedo, J.D.; Maset, M.A.; Segarra N. (2015a): "Formative assessment in groups with a high number of students". En: Proccedings of the 7th International Conference on Education and New Learning Technologies. (Barcelona). Disponible https://library.iated.org/publications/EDULEARN15

CAbedo, J.D.; Maset, M.A.; Segarra N. (2015b): "Evaluación formativa virtual en asignaturas básicas con grupos masificados". En: Univest 2015. (Girona). Disponible en http://www.udg.edu/Portals/9/Publicacions/electroniques/Univest.pdf

GibBS, G.; Simpson, C. (2009). "Condiciones para una evaluación continuada favorecedora delaprendizaje”. Cuadernos de docencia universitaria Num. 13

LARRETA, B. (2006). La coordinación del profesorado ante las demandas del Espacio Europeo de Educación Superior: el caso de la Facultad de Actividad Física y del Deporte de la Universidad Europea de Madrid. Madrid. Ed. Ademas Comunicación.

LÓPez, G.; DE OÑA, R., GARACH, L., CALVO, F.J., DE OÑA, J. (2010): "El nuevo rol del alumno como "grupo profesor" como herramienta para alcanzar algunos de los objetivos perseguidos en Bolonia". En: Actas de las I Jornadas sobre Innovación Docente y Adaptación al EEES. (Granada, p. 153 158). Granada, Godel Impresores Digitales S.L.

LóPEZ, P. (2008). "Desarrollo de competencias en el EEES: evaluación y percepción de alumnos y profesores". En: V Jornadas Internacionales de Innovación Universitaria. (Villaviciosa de Odón, Madrid: UEM).

Ministerio de EduCación, Cultura y Deporte (2010): “La integración del sistema universitario español en el espacio europeo de educación superior" Disponible en www.eees.es/pdf/DocumentoMarco_10_Febrero.pdf

SANZ, J.J. (2011): "Metodología Bolonia y evaluación de competencias en la asignatura de Economía Análisis Matemático”. WP-11-02. European Community Studies Association

(c)) EY-NC-ND 2016, Universitat Politècnica de València 